\title{
REVISED A biochemical logarithmic sensor with broad dynamic
}

\section{range [version 2; peer review: 2 approved]}

\author{
Steven A. Frank \\ Department of Ecology and Evolutionary Biology, University of California, Irvine, CA, 92697-2525, USA
}

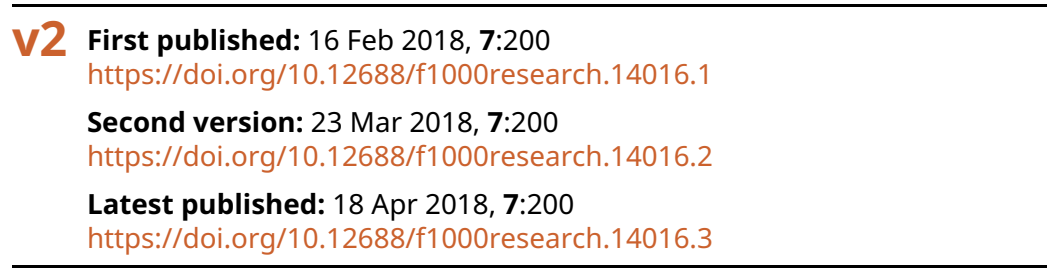

\section{Abstract}

Sensory perception often scales logarithmically with the input level.

Similarly, the output response of biochemical systems sometimes scales logarithmically with the input signal that drives the system. How biochemical systems achieve logarithmic sensing remains an open puzzle. This article shows how a biochemical logarithmic sensor can be constructed from the most basic principles of chemical reactions. Assuming that reactions follow the classic Michaelis-Menten kinetics of mass action or the more generalized and commonly observed Hill equation response, the summed output of several simple reactions with different sensitivities to the input will often give an aggregate output response that logarithmically transforms the input. The logarithmic response is robust to stochastic fluctuations in parameter values. This model emphasizes the simplicity and robustness by which aggregate chemical circuits composed of sloppy components can achieve precise response characteristics. Both natural and synthetic designs gain from the power of this aggregate approach.

\section{Keywords}

Biochemical circuit, Hill equation, synthetic biology, systems biology, aggregation, robustness

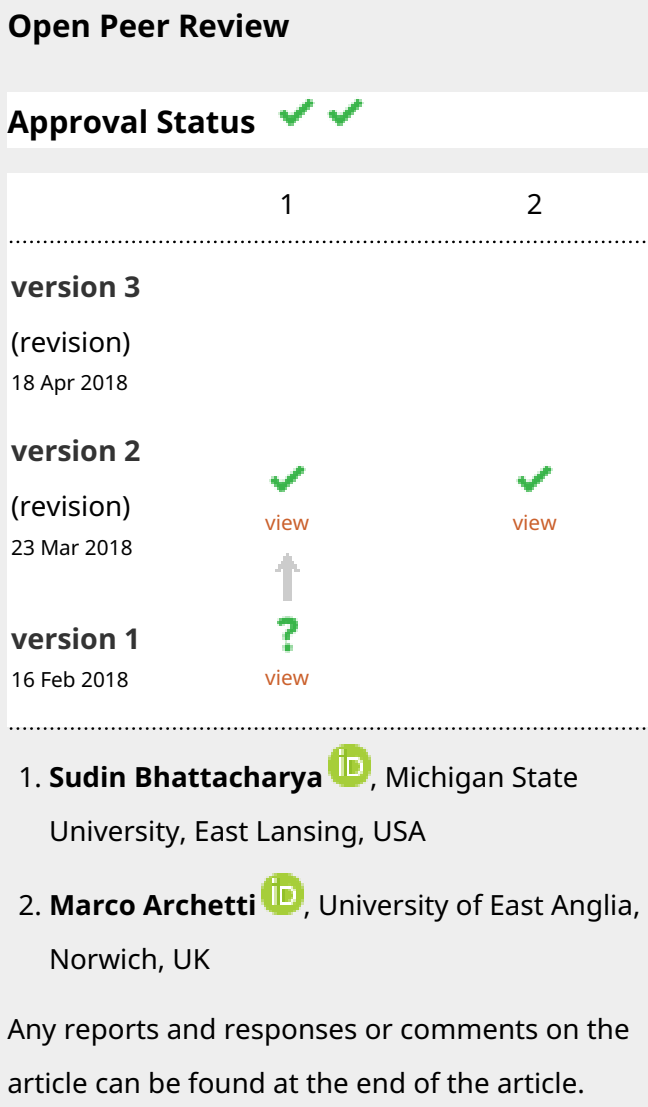


Corresponding author: Steven A. Frank (safrank@uci.edu)

Author roles: Frank SA: Conceptualization, Formal Analysis, Funding Acquisition, Investigation, Methodology, Project Administration, Resources, Software, Supervision, Validation, Visualization, Writing - Original Draft Preparation, Writing - Review \& Editing

Competing interests: No competing interests were disclosed.

Grant information: National Science Foundation grant DEB-1251035 supports my research.

The funders had no role in study design, data collection and analysis, decision to publish, or preparation of the manuscript.

Copyright: @ 2018 Frank SA. This is an open access article distributed under the terms of the Creative Commons Attribution License, which permits unrestricted use, distribution, and reproduction in any medium, provided the original work is properly cited.

How to cite this article: Frank SA. A biochemical logarithmic sensor with broad dynamic range [version 2; peer review: 2 approved] F1000Research 2018, 7:200 https://doi.org/10.12688/f1000research.14016.2

First published: 16 Feb 2018, 7:200 https://doi.org/10.12688/f1000research.14016.1 


\section{REVISED Amendments from Version 1}

I fixed misspelling of Menten's name in "Michaelis-Menten". I have added a Data availability section with the source code used to generate Figure 1.

See referee reports

\section{Introduction}

I present a simple biochemical circuit that logarithmically transforms input signals. This circuit adds the outputs of several reactions that follow standard mass action Michaelis-Menten kinetics. Alternatively, the biochemical kinetics may follow the commonly observed Hill equation response, which includes Michaelis-Menten kinetics as a special case. This sensor has high dynamic range, responding logarithmically across many orders of magnitude. The high dynamic range is achieved by adding together reactions with different sensitivity ranges. The aggregate nature of this circuit provides robustness to parameter variations. Aggregate sensor design may explain the commonly observed high dynamic range of logarithmic biological responses and may also provide a useful tool for synthetic biology.

\section{Results and discussion}

Many biochemical reactions and cellular responses transform an input, $x$, into an output, $y$, according to the Hill equation

$$
y=\frac{x^{k}}{c^{k}+x^{k}},
$$

in which $c$ is the value of the input $x$ that yields one-half of the maximal output response, $k$ is the Hill coefficient that determines the shape of the response, and $y$ is normalized to be between 0 and $1^{1}$. Simple mass action kinetics often follows the Hill equation with $k=1$, which corresponds to classical MichaelisMenten kinetics ${ }^{2}$. For example, the input may drive production of the output, and the output may spontaneously transform back to a prior state.

The output of the Hill equation scales approximately logarithmically with its input through the middle part of its response range, because $y$ is roughly linear with respect to $\log x$. Prior studies have emphasized that a Hill equation response can act as a logarithmic sensor ${ }^{3,4}$. However, a single Hill equation response provides a logarithmic sensor with limited dynamic range (Figure 1A).

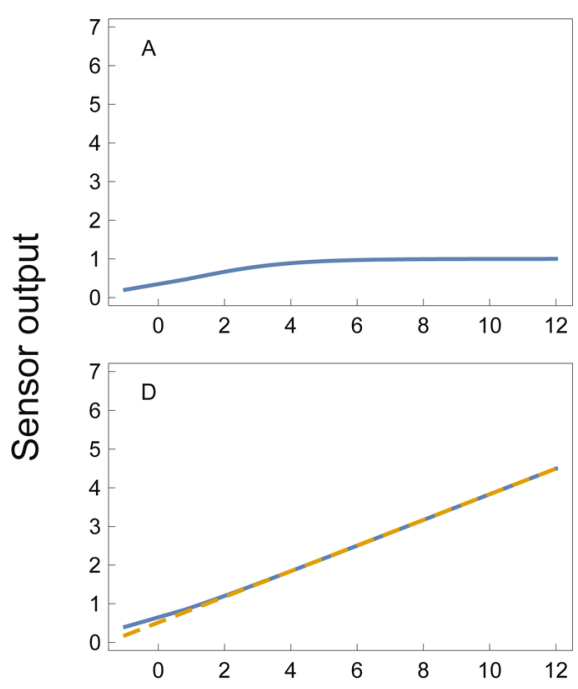

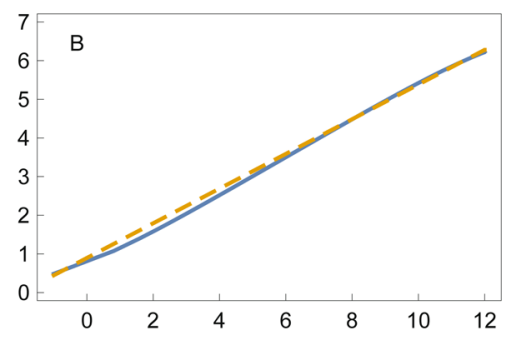

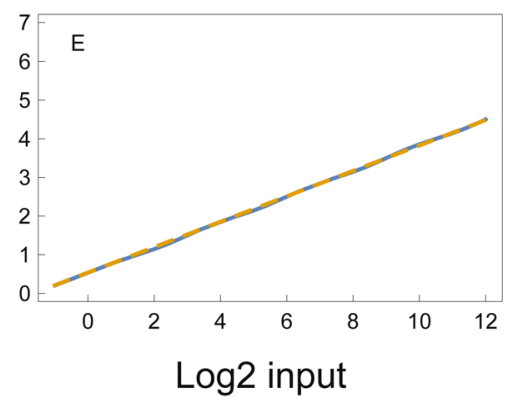

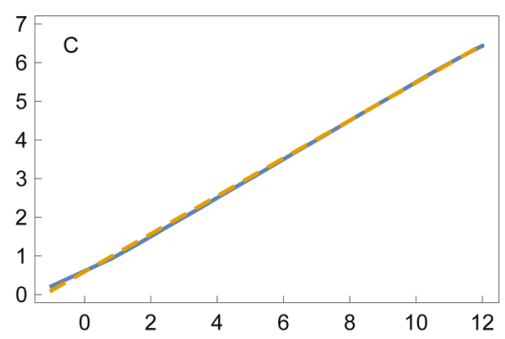

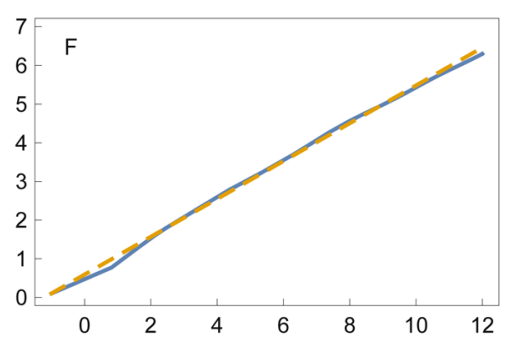

Figure 1. The logarithmic sensor response, $y$, from the sum of Hill equations given in Equation 1, versus the logarithm of the input, $\boldsymbol{x}$, on a $\log _{2}$ scale. Each unit on the input scale corresponds to a doubling of the input. The range of 13 doublings is approximately four orders of magnitude, $2^{13} \approx 10^{4}$. A logarithmic sensor responds linearly with respect to the logarithm of the input. The solid blue lines show the response, $y$, from Equation 1. The dashed gold lines are linear fits to the response. (A) A single Hill equation with $c=1$ and $k=2$. A linear response with low sensitivity occurs over a few doublings at small input levels. The following plots all use $n=7$ and additional parameters as described from the summed Hill equation in Equation 1, in which $c_{i}=b^{i}$. (B) Response with $k=1$ and $b=4$. (C) Response with $k=2$ and $b=$ 4. (D) Response with $k=1$ and $b=8$. (E) Response with $k=2$ and $b=8$. (F) Same average parameters values of $k=2$ and $c_{i}=b^{i}$ for $b=4$ as in the plot above, but with random parameter fluctuations around those average values. For each of the 7 Hill equations in the sum given in Equation 1, each parameter was obtained by a different random number drawn from a normal distribution. For each $k$, the parameters were drawn from a normal distribution with mean 2 and standard deviation $2 \times 0.25=0.5$. For each $c_{i}$, the parameters were drawn from a normal distribution with mean $4^{i}$ and standard deviation $4^{i} \times 0.25$. The response remains nearly linear in spite of the random parameter fluctuations (see Data availability). 
My extended dynamic range sensor arises by adding together $n$ Hill equations with increasing values of the half-maximal response, $c_{i}$, as

$$
y=\sum_{i=0}^{n-1} \frac{x^{k}}{c_{i}^{k}+x^{k}} .
$$

For example, if $c_{i}=b^{i}$, then each reaction in the sum has an increasing input value at which its maximal sensitivity occurs. Figure 1B-E shows that simple combinations of $k$ and $b$ create a logarithmic sensor, in which the output is linearly related to the logarithm of the input. The logarithmic relation holds robustly when the parameters $k$ and $b$ of the individual reactions vary stochastically (Figure 1F).

Several biochemical circuit responses and many aspects of perception scale logarithmically ${ }^{5}$. A robust and generic pattern of this sort seems likely to depend on a robust and generic underlying design. In the search for a generic circuit, my biochemical logarithmic sensor has three advantages over prior designs. First, prior models depended on particular molecular assumptions about biochemical kinetics or reaction pathways ${ }^{3,4}$. My design requires only Michaelis-Menten or Hill equation responses, which are very widely observed in biochemical and cellular systems ${ }^{1}$. Second, prior models focused on single input-output processes, which have relatively narrow dynamic range. My aggregate design provides a logarithmic response over a vastly greater range. Third, the prior models' responses are easily perturbed by parameter fluctuations. My design performs robustly with respect to broad fluctuations in parameters. The robustness of my logarithmic sensor emphasizes the potential to achieve precise response characteristics from underlying sloppy components when using an aggregate design ${ }^{6,7}$.

\section{Data availability}

Mathematica source code to produce Figure 1 can be found at: https://doi.org/10.5281/zenodo. $1203651^{8}$

\section{License: CC BY 4.0}

\section{Competing interests}

No competing interests were disclosed.

Grant information

National Science Foundation grant DEB-1251035 supports my research.

The funders had no role in study design, data collection and analysis, decision to publish, or preparation of the manuscript.
1. Zhang Q, Bhattacharya S, Andersen ME: Ultrasensitive response motifs: basic amplifiers in molecular signalling networks. Open Biol. 2013; 3(4): 130031. PubMed Abstract | Publisher Full Text | Free Full Text

2. Frank SA: Input-output relations in biological systems: measurement, information and the Hill equation. Biol Direct. 2013; 8: 31 PubMed Abstract | Publisher Full Text | Free Full Text

3. Daniel R, Rubens JR, Sarpeshkar R, et al: Synthetic analog computation in living cells. Nature. 2013; 497(7451): 619-623. PubMed Abstract | Publisher Full Text

4. Olsman N, Goentoro L: Allosteric proteins as logarithmic sensors. Proc Natl Acad Sci U S A. 2016; 113(30): E4423-E4430. PubMed Abstract | Publisher Full Text | Free Full Text
5. Adler M, Alon U: Fold-change detection in biological systems. Curr Opin Syst Biol. 2018; 8: 81-89. Publisher Full Text

6. Frank SA: The common patterns of nature. J Evol Biol. 2009; 22(8): 1563-1585. PubMed Abstract | Publisher Full Text | Free Full Text

7. Frank SA: Evolution of robustness and cellular stochasticity of gene expression. PLOS Biol. 2013; 11(6): e1001578. PubMed Abstract | Publisher Full Text | Free Full Text

8. Frank SA: Biochemical logarithmic sensor: Mathematica source code for doi:10.12688/f1000research.14016.1. Zenodo. 2018. Data Source 


\section{Open Peer Review}

\section{Current Peer Review Status:}

\section{Version 2}

Reviewer Report 11 April 2018

https://doi.org/10.5256/f1000research.15653.r32535

(C) 2018 Archetti M. This is an open access peer review report distributed under the terms of the Creative Commons Attribution License, which permits unrestricted use, distribution, and reproduction in any medium, provided the original work is properly cited.

\section{Marco Archetti}

School of Biological Sciences, University of East Anglia, Norwich, UK

This is a brilliant short paper: a clever idea, explained very clearly and that immediately makes sense (but that is by no means obvious at first sight). The revision and the comments about the first version seem appropriate to me.

I don't have further substantial comments except perhaps the following.

I was curious to understand what value of $n$ is required to achieve the result presented in the figure (is there anything special about $n=7$ ?). Using the code provided this is easy to check, but perhaps it would help readers to add an additional figure with multiple curves for values of $n$ ranging between 1 and 7 to see how the results change. Doing this exercise myself I learned, for instance, that $n<7$ is enough for certain parameters and that the value of $b$ is especially important (something that perhaps could be discussed in a sentence or two).

Is the work clearly and accurately presented and does it cite the current literature? Yes

Is the study design appropriate and is the work technically sound? Yes

Are sufficient details of methods and analysis provided to allow replication by others? Yes

If applicable, is the statistical analysis and its interpretation appropriate? Not applicable

Are all the source data underlying the results available to ensure full reproducibility? Yes 
Are the conclusions drawn adequately supported by the results?

Yes

Competing Interests: No competing interests were disclosed.

I confirm that I have read this submission and believe that I have an appropriate level of expertise to confirm that it is of an acceptable scientific standard.

Author Response ( F1000Research Advisory Board Member) 12 Apr 2018

Steven Frank, Department of Ecology \& Evolutionary Biology, University of California, Irvine, USA

I thank Dr. Archetti for his suggestion to illustrate the response over various values of the parameters $n$ and $b$. In the new revision of the article, I have added Figures 2 and 3 to show the sensor's response over a broad range of these parameters.

Competing Interests: No competing interests were disclosed.

Reviewer Report 04 April 2018

https://doi.org/10.5256/f1000research.15653.r32373

(C) 2018 Bhattacharya S. This is an open access peer review report distributed under the terms of the Creative Commons Attribution License, which permits unrestricted use, distribution, and reproduction in any medium, provided the original work is properly cited.

\section{Sudin Bhattacharya}

Department of Biomedical Engineering, Department of Pharmacology \& Toxicology, Institute for Quantitative Health Science and Engineering, Michigan State University, East Lansing, MI, USA

I am satisfied with the author's response to my comments about his article, which at this stage is a theoretical model of how a natural or artificial biochemical logarithmic sensor might work.

Is the work clearly and accurately presented and does it cite the current literature? Yes

Is the study design appropriate and is the work technically sound? Yes

Are sufficient details of methods and analysis provided to allow replication by others? Yes

If applicable, is the statistical analysis and its interpretation appropriate? Yes 
Are all the source data underlying the results available to ensure full reproducibility? Yes

Are the conclusions drawn adequately supported by the results?

Yes

Competing Interests: No competing interests were disclosed.

Reviewer Expertise: Computational biology and toxicology

I confirm that I have read this submission and believe that I have an appropriate level of expertise to confirm that it is of an acceptable scientific standard.

\section{Version 1}

Reviewer Report 19 March 2018

https://doi.org/10.5256/f1000research.15234.r30929

(C) 2018 Bhattacharya S. This is an open access peer review report distributed under the terms of the Creative Commons Attribution License, which permits unrestricted use, distribution, and reproduction in any medium, provided the original work is properly cited.

\section{Sudin Bhattacharya}

Department of Biomedical Engineering, Department of Pharmacology \& Toxicology, Institute for Quantitative Health Science and Engineering, Michigan State University, East Lansing, MI, USA

The author presents an interesting model of a robust biochemical logarithmic switch with a broad dynamical range as a sum of multiple Hill-equation based models.

1. My main problem with this work is the lack of a biological context with a real example (the author argues the model applies to natural as well as artificial circuits). Is the author proposing that the successive Hill-type switches would be arranged in series? A more natural example would be a layered cascade like a MAP kinase cascade ${ }^{1}$. But in that case the output would not be a simple linear sum as in the author's Eqn. (1), but rather something like a Hill function of a Hill function.

2. The contention that "My design performs (more) robustly with respect to broad fluctuations in parameters" should be established more rigorously. How were the stochastic simulations performed? It's hard to tell since no methodological details are provided.

3. "Menten" in Michaelis-Menten mis-spelt throughout manuscript (after Maud Menten: https://en.wikipedia.org/wiki/Maud_Menten)

\section{References}


1. Huang CY, Ferrel JE: Ultrasensitivity in the mitogen-activated protein kinase cascade. Proceedings of the National Academy of Sciences. 1996; 93: 10078-10083

Is the work clearly and accurately presented and does it cite the current literature? Yes

Is the study design appropriate and is the work technically sound?

No

Are sufficient details of methods and analysis provided to allow replication by others? Partly

If applicable, is the statistical analysis and its interpretation appropriate? Partly

Are all the source data underlying the results available to ensure full reproducibility? Yes

Are the conclusions drawn adequately supported by the results? No

Competing Interests: No competing interests were disclosed.

Reviewer Expertise: Computational biology and toxicology

I confirm that I have read this submission and believe that I have an appropriate level of expertise to confirm that it is of an acceptable scientific standard, however I have significant reservations, as outlined above.

Author Response ( F1000Research Advisory Board Member) 19 Mar 2018

Steven Frank, Department of Ecology \& Evolutionary Biology, University of California, Irvine, USA

I thank Dr. Bhattacharya for sharing his expertise on topics related to the important Hill equation (see ref. 1 of the article). Dr. Bhattacharya made three helpful comments in his review, which I respond to in reverse order.

First, I appreciate the spelling correction. I had reread the interesting history of the Michaelis-Menten equation when preparing this article, but nonetheless incorrectly spelled Menten's name. I have fixed that misspelling in the revision.

Second, I have uploaded the source code, as a supplementary file, that I used to do the stochastic simulations. The short, simple code provides a clear, exact description of the methods. The code was used to generate Figure 1, and can be used to regenerate that figure and explore alternative assumptions. A summary of the methods are provided in the legend for Figure 1. 
The final point concerns the lack of specific biological examples and details. The first aspect of this comment is a question asked by Dr. Bhattacharya: "Is the author proposing that the successive Hill-type switches would be arranged in series?"

I am proposing a summation of individual responses to a stimulus, as described in Equation 1. For example, suppose that a set of sensors respond to sound intensity. Each sensor has a Hill-like response curve, but tuned to different intensity level. The output of the aggregate set of sensors is the sum of the individual sensor responses. Each sensor may comprise a simple or complex set of reactions. All that we care about here is that the net response per sensor is Hill-like in shape.

That same logic could describe the response to a molecular signal. Various reactions could each have a Hill equation response, but tuned to different sensitivities. The aggregate consequence of those responses would be a net output level.

With regard to an explicit example, I gave a lot of thought to that issue while preparing the manuscript. I could not find a compelling example for which sufficient biological detail about mechanism had been described. There are plausible cases that are worth exploring, such as the sensory problems that I mentioned. For molecular systems, there are hormones for which cells have multiple receptors, presumably tuned differently with regard to response and other aspects (e.g., estrogen receptors). However, I agree that those multireceptor systems do not provide a compelling case given current knowledge.

Why are there no compelling molecular examples? I think the reason is that researchers rarely look for responses that arise by aggregate processes, such as summation of underlying reactions. Instead, the search is almost always for a single reaction or explicit pathway that exactly matches the desired input-output pattern. So my goal for this article is to suggest a plausible alternative for consideration.

One advantage of publishing with F1000Research is that I can update the article in the future. If a good example arises in discussion with readers of the article or from the literature, I will update the article to include that example. For now, I think it is most important that the novel idea be published in a concise and direct manner, with emphasis on the simple logic of the model.

Competing Interests: No competing interests were disclosed. 
The benefits of publishing with F1000Research:

- Your article is published within days, with no editorial bias

- You can publish traditional articles, null/negative results, case reports, data notes and more

- The peer review process is transparent and collaborative

- Your article is indexed in PubMed after passing peer review

- Dedicated customer support at every stage

For pre-submission enquiries, contact research@f1000.com 